\title{
Modelling the cost-effectiveness of pay-for- performance in primary care in the UK
}

\author{
Ankur Pandya ${ }^{1,3^{*}}$, Tim Doran $^{2}$, Jinyi Zhu ${ }^{3}$, Simon Walker ${ }^{4}$, Emily Arntson ${ }^{5}$ and Andrew M. Ryan ${ }^{5}$
}

\begin{abstract}
Background: Introduced in 2004, the United Kingdom's (UK) Quality and Outcomes Framework (QOF) is the world's largest primary-care pay-for-performance programme. Given some evidence of the benefits and the substantial costs associated with the QOF, it remains unclear whether the programme is cost-effective. Therefore, we assessed the costeffectiveness of continuing versus stopping the QOF.

Methods: We developed a lifetime simulation model to estimate quality-adjusted life years (QALYs) and costs for a UK population cohort aged 40-74 years $(n=27,070,862)$ exposed to the QOF and for a counterfactual scenario without exposure. Based on a previous retrospective cross-country analysis using data from 1994 to 2010, we assumed the benefits of the QOF to be a change in age-adjusted mortality of -3.68 per 100,000 population (95\% confidence interval -8.16 to 0.80 ). We used cost-effectiveness thresholds of $£ 30,000 / \mathrm{QALY}, £ 20,000 / \mathrm{QALY}$ and $£ 13,000 / \mathrm{QALY}$ to determine the optimal strategy in base-case and sensitivity analyses.

Results: In the base-case analysis, continuing the QOF increased population-level QALYs and health-care costs yielding an incremental cost-effectiveness ratio (ICER) of $£ 49,362 / Q A L Y$. The ICER remained $>£ 30,000 / Q A L Y$ in scenarios with and without non-fatal outcomes or increased drug costs, and under differing assumptions about the duration of QOF benefit following its hypothetical discontinuation. The ICER for continuing the programme fell below £30,000/QALY when QOF incentive payments were 36\% lower (while preserving QOF mortality benefits), and in scenarios where the QOF resulted in substantial reductions in health-care spending or non-fatal cardiovascular disease events. Continuing the QOF was cost-effective in 18\%, 3\% and $0 \%$ of probabilistic sensitivity analysis iterations using thresholds of $£ 30,000$ / QALY, $£ 20,000 / Q A L Y$ and $£ 13,000 / Q A L Y$, respectively.

Conclusions: Compared to stopping the QOF and returning all associated incentive payments to the National Health Service, continuing the QOF is not cost-effective. To improve population health efficiently, the UK should redesign the QOF or pursue alternative interventions.
\end{abstract}

\section{Background}

Introduced in 2004, the United Kingdom's Quality and Outcomes Framework (QOF) is the world's largest primary-care pay-for-performance programme. The QOF links up to $25 \%$ of general practitioners' income to performance on a wide range of quality indicators related to clinical management of common chronic conditions, organisation of care and patient experience [1]. This supplements existing payments to practices, which are

\footnotetext{
* Correspondence: anpandya@hsph.harvard.edu

'Department of Health Policy and Management, Harvard T.H. Chan School of Public Health, 718 Huntington Ave, 2nd Floor, Boston, MA 02115, USA

${ }^{3}$ Center for Health Decision Science, Harvard T.H. Chan School of Public Health, Boston, MA, USA

Full list of author information is available at the end of the article
}

largely provided through capitation payments. Research on the QOF suggests that the programme accelerated improvement for the incentivised indicators in the 3 years following its implementation [2]. However, this improvement appeared to attenuate over time [3-5]. A recent analysis also found that the QOF did not significantly improve mortality for disease areas targeted under the programme [6].

The QOF is subject to annual review, with changes agreed in negotiations between National Health Service (NHS) Employers and the British Medical Association's General Practitioners Committee, informed by indicator development work conducted by the National Institute for Health and Care Excellence. In 2014/15, 40 indicatorsaccounting for $35 \%$ of the value of total incentive

(c) The Author(s). 2018 Open Access This article is distributed under the terms of the Creative Commons Attribution 4.0 International License (http://creativecommons.org/licenses/by/4.0/), which permits unrestricted use, distribution, and reproduction in any medium, provided you give appropriate credit to the original author(s) and the source, provide a link to the Creative Commons license, and indicate if changes were made. The Creative Commons Public Domain Dedication waiver (http://creativecommons.org/publicdomain/zero/1.0/) applies to the data made available in this article, unless otherwise stated. 
payments-were removed from the scheme without replacement, with most of the associated resource used to increase capitation payments [7]. In 2016/17, QOF was discontinued altogether in Scotland and funding was transferred to capitation payments [8]. QOF continues in England, Wales and Northern Ireland, although options for reform or replacement are being considered.

Despite the large costs of the QOF and other pay-forperformance programmes, almost no evidence exists on their cost-effectiveness and how this compares to other system-level interventions to improve longevity $[5,9,10]$. Pay-for-performance programmes introduce additional economic costs to the health-care system, which could have been spent on other health interventions or policies. A cost-effectiveness analysis, the standard method for assessing value for money, can be used to determine whether additional spending on pay-for-performance is worth the health gains produced by these policies. Although a cost-effectiveness analysis has previously been conducted for other pay-for-performance programmes in the UK, decisions on the development or discontinuation of QOF have not been informed by reliable estimates of cost-effectiveness [11]. A systematic review of pay-for-performance programmes found that, despite the promise of cost-effective financial incentives, convincing evidence of the cost-effectiveness of pay-forperformance was lacking [12]. Previous attempts to estimate the cost-effectiveness of pay-for-performance have extrapolated evidence from randomised trials, rather than using direct evidence of its effectiveness on outcomes $[4,9,13]$. These approaches are limited because results from randomised trials may not generalise to the older, sicker patients who are typically excluded from trials [6].

In this study, we address this knowledge gap by evaluating the cost-effectiveness of the QOF under various assumptions around the programme's benefits and costs.

\section{Methods \\ Overview}

We developed a computer-based simulation model to estimate the QOF-related lifetime health effects and costs for a representative UK general population cohort aged $40-74$ years $(n=27,070,862)$ exposed to two competing policy alternatives: (1) continuing the QOF and (2) stopping the QOF. We compared the trade-off between lifetime discounted health effects, quantified using quality-adjusted life years (QALYs), and QOF-related health-care costs using an incremental cost-effectiveness analysis from a NHS (health-care payer) perspective. The simulation model discounts health effects and costs using a rate of 3.5\% [14]. An overview of our methodology is shown in Fig. 1.

\section{Simulation model and population}

The overall goal for our model-based analysis was to estimate the impact of continuing the QOF (compared to stopping the QOF) on lifetime QALYs and costs. The decision of whether or not to continue the QOF will have the most impact on the specific diseases targeted by the QOF. For instance, the lifetime QALYs gained would be greater if the QOF mortality reductions were concentrated in healthy children as opposed to older persons with multiple chronic conditions. Of all the conditions actually included in the QOF, cardiovascular diseases and associated risk conditions (coronary heart disease, stroke, diabetes and hypertension) collectively attract the largest incentives and account for the majority of non-cancer deaths (although cancer is included in the QOF, it only receives weak incentives) [6, 15-17]. In 2005 , payments of up to $£ 15,125$ were available for the average family practice across 15 ischaemic heart disease and heart failure indicators, but only $£ 1500$ was available across two cancer indicators [6]. Therefore, we assumed that the QOF benefits were concentrated in individuals with cardiovascular disease (aged 40-

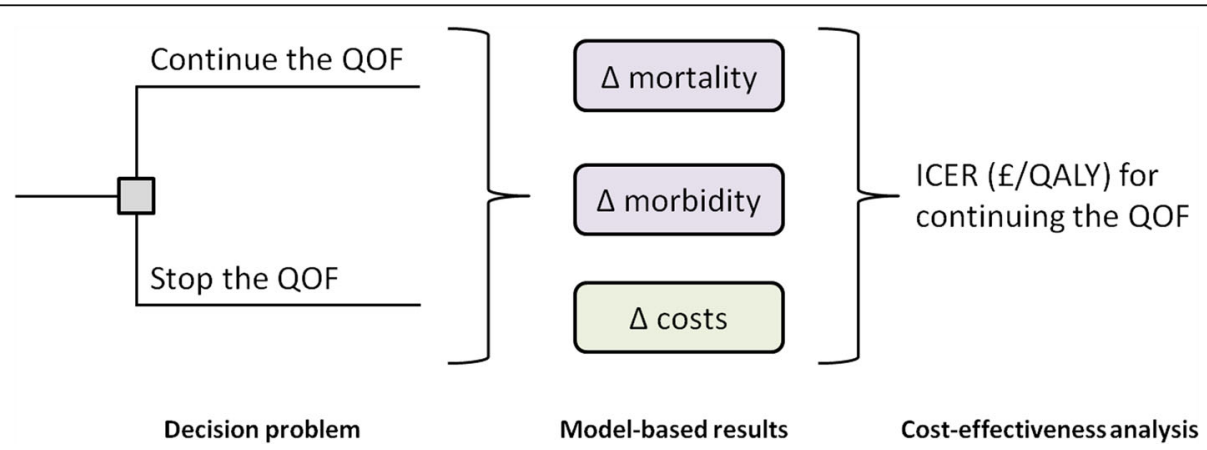

Fig. 1 Conceptual diagram of the cost-effectiveness analysis. Individuals enter the simulation model and are assigned to one of two QOF scenarios. The model estimates the impact of continuing or stopping the QOF on mortality, morbidity and QOF-related cost outcomes. The trade-offs between quality-adjusted life years (QALYs) and costs are evaluated by calculating an incremental cost-effectiveness ratio (ICER) for continuing the QOF. ICER incremental cost-effectiveness ratio, QALY quality-adjusted life year, QOF Quality and Outcomes Framework 
74 years at baseline) in our base-case analysis, but relaxed this assumption in sensitivity analyses (including a scenario where the QOF equally benefited all individuals aged 0-74 years with and without disease). We needed to make these assumptions around which population segments would benefit from the QOF because our main effectiveness estimate for the QOF did not specify in whom the mortality benefits were concentrated, but rather reported an overall age-adjusted mortality reduction of 3.68 per 100,000 (across those aged 0-74 years) [6].

We created a hypothetical cohort that would be affected by the QOF using age group- and sex-specific population sizes for the UK, further dividing this population into those with and those without prevalent cardiovascular disease. We used Office for National Statistics data for sex-specific population sizes in 5-year age categories (e.g., 2,255,562 females aged 50-54 years at baseline) and sex- and age-specific cardiovascular disease prevalence estimates from British Heart Foundation data (e.g. $8.4 \%$ of females aged $50-54$ years at baseline had prevalent cardiovascular disease, ICD-10 codes I00-I99) [15, 16]. Our model uses annual cycles to project the life expectancy, quality-adjusted life expectancy and QOF-related costs for a representative UK cohort until death. Each model population segment faces a risk of death in each model cycle based on the sex, age and cardiovascular disease status of the group being simulated, with additional mortality risk adjustments to account for the absence of the QOF for the non-QOF strategy (i.e. the current life tables include the effects of the QOF, so we increased the risk of death in the life tables when simulating the non-QOF strategy). Those with cardiovascular disease faced higher mortality risks and reduced quality of life compared to those without cardiovascular disease. To estimate mortality risks for those with cardiovascular disease, we multiplied the annual mortality risk all-cause life tables (i.e. the age-specific annual risks of death from any cause) by 1.6 (for men) or 2.1 (for women). These age-adjusted mortality ratios were based on an analysis of linked hospital discharge and 7-year mortality follow-up data from survivors of first acute myocardial infarction in England from 2004 to 2010 [18]. We subtracted cardiovascular disease mortality rates from all-cause life tables to estimate mortality risks for those without cardiovascular disease [18]. The model sums all health effects and costs accrued for each population segment, yielding populationlevel results. Table 1 shows all model inputs and data sources. The model is programmed in Microsoft Excel with Visual Basic for Applications and is included as Additional file 1.

\section{QOF mortality effects}

The mortality increase from stopping the QOF was estimated from a previous difference-in-difference analysis [6]. In their study, Ryan et al. created a synthetic control group as a weighted combination of countries previously characterised as having a high-income epidemiological profile. Based on their study, the QOF programme was associated with a change in age- and sex-adjusted mortality from the major contributors to population mortality targeted by the QOF (ischaemic heart disease, hypertension, stroke, diabetes, chronic kidney disease, asthma and chronic pulmonary disease) of -3.68 per 100,000 population ( $95 \%$ confidence interval of -8.16 to 0.80 ).

Table 1 Model variables with base-case values and ranges used in sensitivity analyses

\begin{tabular}{|c|c|c|c|}
\hline Variable & Base-case value & Sensitivity analysis range & Source(s) \\
\hline QOF mortality benefit (age- and sex-adjusted per 100,000) & -3.68 & -8.16 to 0.80 & {$[6]$} \\
\hline Adjusted QOF mortality benefit (for those with age $>40$ years with CVD) & -58.93 & -130.57 to 12.81 & Calculated \\
\hline All-cause age- and sex-specific mortality (and age and sex demographics) & Life table & Not applicable & {$[45]$} \\
\hline CVD prevalence, males (aged $45-64$ years, aged $65-74$ years) & $14.6 \%, 28.5 \%$ & $+/-20 \%$ & {$[16]$} \\
\hline CVD prevalence, females (aged 45-64 years, aged 65-74 years) & $8.4 \%, 22.5 \%$ & $+/-20 \%$ & {$[16]$} \\
\hline CVD mortality multiplier (male, female) & $1.6,2.1$ & $+/-20 \%$ & {$[18]$} \\
\hline CVD utility & 0.796 & $+/-20 \%$ & {$[21]$} \\
\hline Non-fatal-to-fatal CVD events averted (ratio) & 1.63 & $0-10$ & {$[20]$} \\
\hline QOF annual population-level incentive costs & $£ 1,396,843,151$ & $£ 0-2,000,000,000$ & $\begin{array}{l}\text { Country-specific } \\
\text { sources }[24,26,27]\end{array}$ \\
\hline QOF effect on utilisation costs per $£$ spent on incentives & $£ 0.011$ & $-£ 1-£ 1$ & {$[29,30]$} \\
\hline Acute CVD event costs (i.e. costs within first year of CVD event) & $£ 10,871$ & $+/-20 \%$ & {$[31]$} \\
\hline Chronic CVD event costs (i.e. annual costs for all years after first year) & $£ 3282$ & $+/-20 \%$ & [31] \\
\hline Average NHS costs by age & Age-based table & $£ 0$ to $+100 \%$ & {$[32]$} \\
\hline Discount rate & $3.5 \%$ & $0-5 \%$ & [14] \\
\hline
\end{tabular}

QOF Quality and Outcomes Framework, CVD cardiovascular disease, NHS National Health Service 
This estimate was based on age-adjusted mortality rates for populations aged 0-74 years. Since QOF mortality benefits would be concentrated in older individuals with prevalent disease, we adjusted the mortality effect used in our model by back-calculating mortality change (58.9 per 100,000 population) in older individuals (aged 40-74 years, $n=27,070,862$ ) with prevalent cardiovascular disease that would result in the Ryan et al. general population result. In other words, a 58.9 per 100,000 mortality decrease concentrated exclusively in individuals aged $40-74$ years with cardiovascular disease would result in a 3.68 per 100,000 mortality effect in all individuals (aged $0-74$ years among individuals with and without cardiovascular disease, $n=59,385,341)$. We performed a sensitivity analysis using a 3.68 per 100,000 mortality decrease for all individuals (aged 0-74 years) to assess the impact of these two contrasting assumptions (mortality effect concentrated in those aged 40-74 years versus mortality effect spread out among those aged 0-74 years) on the model results. While either assumption had QOF mortality effects stopping at age 74 years (due to our data source [6]), the model cohort was modelled until death in all analyses.

In the base-case analyses, we assumed the QOF-related mortality benefit was immediately lost if the QOF were discontinued. In the sensitivity analyses, we modelled different durations over which the mortality benefit of the QOF waned if the programme were discontinued. In these scenarios, we assumed linear declines in the QOF mortality benefit from first year in the model to a time in the future $(1,3,5$ or 10 years from the model start), at which point the mortality benefit from the QOF would equal zero.

\section{QOF non-fatal effects (i.e. QOF morbidity effects)}

Best practice in a cost-effectiveness analysis is to include all health outcomes (fatal and non-fatal) that differ from the choice of a particular strategy over another [19]. While it is likely that the QOF resulted in changes in both fatal and non-fatal outcomes, the Ryan et al. study did not include non-fatal outcomes due to data restrictions. Given the QOF focus on managing cardiovascular disease risk, we applied a ratio of non-fatal cardiovascular disease events averted for every fatal event averted from the QOF based on prevented cardiovascular disease events from a meta-analysis of statin trial data from 90,056 participants [20]. First, we calculated the differences in non-fatal coronary heart disease events between statin and placebo arms in the meta-analysis $(1789-2460)$ and divided that difference by the difference in coronary heart disease deaths (1548 - 1960), which resulted in a ratio of nonfatal-to-fatal coronary heart disease events averted of 1.63. We multiplied this ratio by the difference in mortality between the QOF and non-QOF counterfactuals in the model to estimate the number of non-fatal cardiovascular disease events averted by the QOF in each model cycle. The model incorporates morbidity in the QALY calculation by applying utility values (see Health-related quality-of-life section below) to the non-fatal cardiovascular disease events averted by the QOF. We performed sensitivity analyses excluding non-fatal health effects attributable to the QOF in the model in addition to varying the value of the non-fatal-to-fatal ratio used in the model.

\section{Health-related quality of life}

The main effectiveness measure for our analysis was lifetime cohort-level QALYs. The model calculates QALYs by multiplying the number of individuals alive in a given year by a utility value, which quantifies morbidity. The model assigns utility values ranging between 0 (death) and 1 (perfect health) for each year based on age and cardiovascular disease status. Older individuals and those with cardiovascular disease have lower utility values than younger individuals and those without cardiovascular disease, respectively. Utility values were estimated from the results of the EuroQOL 5 Dimensions (EQ-5D) questionnaire from the Medical Expenditure Panel Survey (US-based descriptive responses to the EQ-5D were combined with UK community-based preference weights to calculate utility values for the UK) [21-23]. For a given age (40-100 years), population segments with cardiovascular disease were assigned the minimum utility for cardiovascular disease (0.796) and age-based utilities (ranging from 0.691-0.848). Population segments without cardiovascular disease were assigned the age-based utility values [23].

\section{QOF costs}

We included two major QOF-related cost categories: (1) incentive costs and (2) costs related to changes in health-care utilisation as a result of QOF incentives. We estimated total incentive costs over the first 7 years of the programme at $£ 1,396,843,151$ per year based on remuneration data from the four constituent countries of the UK (see Table 4 in the Appendix) [24-27]. These costs include payments for all domains (clinical, organisational and patient experience) and are considered incremental (i.e. new) costs to the health system, as opposed to reallocating existing funds within the health system, as they represent additional resources the UK government committed to primary care in 2004 [28].

Given the focus of the QOF incentives on disease management in the cardiovascular domain, we assumed changes in health-care utilisation driven by the QOF would be concentrated in the utilisation of cardiovascular disease drugs (targeting cholesterol, blood pressure and diabetes). We estimated changes in drug utilisation based on an observational study in Scotland, which reported an average increase in defined daily doses (DDDs) per prescribing unit (PU) per month of QOF-related drugs 
of 3.79. Among these drugs, cardiovascular disease drugs accounted for 3.47 of the average increase (DDD per PU, $89 \%$ of the total increase across all drugs mentioned or implied by the QOF) [29]. For these costs, we used separated DDD per PU per month estimates from that analysis for five cardiovascular disease drug classes (lipid-regulating, renin-angiotensin system, thiazides and related diuretics, oral antidiabetics and antiplatelets) in conjunction with weighted averages of drug class prices (Table 5 in the Appendix) to estimate an overall annual increase in QOF-related drug costs of $£ 15,692,470$ (i.e. a ratio of 0.011 of increased drug utilisation per $£ 1$ spent on incentives) [30]. We allowed this value to be negative (i.e. a cost saving) in a sensitivity analysis to evaluate the possibility that QOF incentives might result in a net decrease in overall health-care spending from averted disease events and follow-up spending, by varying the ratio from -1 to 1 in a one-way sensitivity analysis. In the model, averted cardiovascular disease events (fatal and non-fatal) resulted in cost savings from averted health-care utilisation. We estimated the costs of averted cardiovascular disease events in the first year of the event $(£ 10,871)$ and all subsequent years $(£ 3282)$ from a previous analysis of linked UK cohort datasets with utilisation and cost data [31]. We modelled future average age-based annual health-care costs based on English NHS data and performed separate sensitivity analyses where these costs were excluded and doubled [32]. All costs in our analyses are reported in 2016 pounds sterling $(£)$.

\section{Base-case cost-effectiveness analysis}

We used conventional incremental cost-effectiveness analysis methods to calculate an incremental cost-effectiveness ratio (ICER) for continuing the QOF programme compared to stopping the QOF. We used cost-effectiveness thresholds of $£ 30,000 / Q A L Y, £ 20,000$ / QALY and $£ 13,000 /$ QALY. These thresholds are based on current National Institute for Health and Care Excellence recommendations (£20,000-30,000/QALY) and a 2015 Claxton et al. study that estimated an empirically based opportunity cost for health-care spending (i.e. a cost-effectiveness threshold that estimates the amount of money, at the margin of health-care spending, it would take to produce one additional QALY) in the UK ( $£ 13,000 / \mathrm{QALY})$ [33]. We also calculated the opportunity costs of continuing the QOF in terms of population-level incremental net health benefit, which converts incremental costs into QALYs using a cost-effectiveness threshold and subtracts these opportunity costs from QALYs gained as a result of continuing the QOF. In other words, the incremental net health benefit captures the health gains to individuals who benefit from funding being given to an intervention minus the population health forgone as a result of committed resources being unavailable for other individuals' health care. The health forgone by other individuals was estimated based on the cost-effectiveness thresholds noted above [specifically, the QALYs forgone (incremental net health benefit) equal the incremental costs divided by the cost-effectiveness threshold] [34].

\section{Sensitivity analyses}

We performed four types of sensitivity analyses: (1) scenario analyses for the inclusion of non-fatal health effects and increased drug utilisation as a result of the QOF and the duration of the QOF mortality benefit if the QOF were discontinued, (2) one-way sensitivity analyses with high and low values for all model inputs, (3) a two-way sensitivity analysis for different combinations of the levels of QOF incentive payments and the QOF mortality benefit and (4) a probabilistic sensitivity analysis, where we assessed the statistical uncertainty in the QOF mortality benefit (which was based on an estimate that had a $95 \%$ confidence interval of -0.80 to 8.16 ) by drawing 1000 random values for the QOF mortality benefit from a normal probability distribution [35]. The probabilistic sensitivity analysis results for each of the 1000 iterations were used to construct a cost-effectiveness acceptability curve showing the probability that continuing the QOF was cost-effective while varying the cost-effectiveness threshold from $£ 0-200,000 / \mathrm{Q} A L Y$.

\section{Results}

\section{Base-case results}

Continuing the QOF programme indefinitely would result in more life years, QALYs and QOF-related costs compared to stopping the QOF (Table 2). Compared to stopping the QOF, continuing the QOF had an ICER of $£ 49,362 / \mathrm{QALY}$ under base-case assumptions (non-fatal outcomes and increased drug costs included, with instant changes in the QOF mortality benefit if the QOF is discontinued, Table 2). The population opportunity cost of continuing the QOF (i.e. the incremental net health benefit) ranged from 226,109 to 979,917 QALYs lost, assuming cost-effectiveness thresholds of $£ 30,000$ /QALY to $£ 13,000$ / QALY, respectively (Table 6 in the Appendix).

\section{Scenario analyses}

Table 3 shows the matrix of ICERs for the QOF for every combination of assumptions related to the inclusion of non-fatal outcomes, the inclusion of increased drug costs and waning of the QOF benefit. The ICER remained greater than $£ 30,000 / Q A L Y$ for every combination of assumptions. The ICER for continuing the QOF was $£ 42,296 /$ QALY when we assumed all individuals aged 40-74 years experienced the health benefits of the QOF (as opposed to only those with prevalent cardiovascular disease), and $£ 31,089 / \mathrm{QALY}$ when the benefits of the QOF were spread out to all individuals (with and 
Table 2 Base-case population-level results and incremental cost-effectiveness analysis of continuing the QOF vs. stopping the QOF

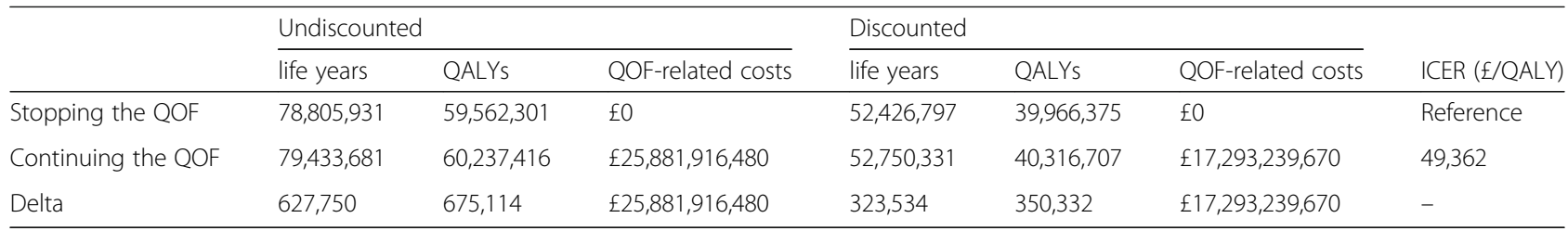

QOF Quality and Outcomes Framework, QALY quality-adjusted life year, ICER incremental cost-effectiveness ratio

without disease) aged 0-74 years (these findings can be reproduced using the simulation model attached as Additional file 1).

\section{One-way sensitivity analyses}

The ICER was only below alternative National Institute for Health and Care Excellence thresholds of $£ 30,000 /$ QALY, $£ 20,000 /$ QALY and $£ 13,000 /$ QALY when, for every $£ 1$ spend on QOF incentives, $£ 0.36, £ 0.55$ or $£ 0.68$ were saved as a result of net cost reductions (for example, from averted cardiovascular disease events; Figure 4 in the Appendix). Similarly, the ICER was below the thresholds of $£ 30,000 / Q A L Y, £ 20,000 / Q A L Y$ and $£ 13,000 / Q A L Y$ when the ratios for non-fatal-to-fatal cardiovascular disease events averted were 3.7 (225\% of the base-case value), 5.5 (335\% of the base-case value) and 7.3 (450\% of the base-case value, Figure 5 in the Appendix).

The cost-effectiveness results were robust (ICERs within $£ 40,000-65,000 / Q A L Y$ ) in the following sensitivity analyses (with high and low parameter ranges as reported in Table 1, unless otherwise specified): applying the QOF mortality benefit to all individuals aged 0-74 years as opposed to a concentrated (higher) mortality benefit to those with prevalent cardiovascular disease, high and low utility values, high and low cardiovascular disease prevalence and mortality multiplier estimates, and separate scenarios removing and doubling the annual average future health-care costs (Table 7 in the Appendix).

\section{Two-way sensitivity analysis}

Fig. 2 shows the ICER as a function of the levels of QOF incentive payments and the QOF mortality benefit assuming a cost-effectiveness threshold of $£ 30,000 / \mathrm{QALY}$. The ICER was below thresholds of $£ 30,000 / Q A L Y$, $£ 20,000 / \mathrm{QALY}$ and $£ 13,000 / \mathrm{QALY}$ with QOF incentive payment levels of $£ 893,979,617$ (64\% of the base-case value), $£ 628,579,418(45 \%)$ and $£ 446,989,808$ (32\%), respectively. Similarly, the ICER was below thresholds of $£ 30,000 / \mathrm{QALY}, £ 20,000 / \mathrm{QALY}$ and $£ 13,000 / \mathrm{QALY}$ with QOF age-adjusted mortality reductions of 5.89 (160\% of the base-case value), 8.25 (225\%) and 11.59 (315\%) respectively.

\section{Probabilistic sensitivity analyses}

Fig. 3 shows the cost-effectiveness acceptability curve from the probabilistic sensitivity analysis. The QOF was cost-effective in $18.3 \%, 2.9 \%$ and $0.1 \%$ of probabilistic sensitivity analysis iterations using thresholds of $£ 30,000 / \mathrm{QALY}, £ 20,000 / \mathrm{QALY}$ and $£ 13,000 / \mathrm{Q} A L Y$, respectively. The $95 \%$ credible interval (i.e. the range where $95 \%$ of probabilistic sensitivity analysis results fell) for the ICER was $£ 19,700 /$ QALY to QOF dominated (which means continuing the QOF would result in higher costs and fewer QALYs compared to stopping the QOF).

\section{Discussion}

In this study we modelled the cost-effectiveness of continuing the QOF in the UK to evaluate whether the incremental health gains from continuing the pay-forperformance programme would be worth the additional costs to do so. We found that the ICER for continuing the QOF was $£ 49,362 / \mathrm{QALY}$, with an $18 \%$ probability of being cost-effective in probabilistic sensitivity analysis

Table 3 Cost-effectiveness ratios (£/QALY) for continuing the QOF versus stopping the QOF under various model scenarios

\begin{tabular}{|c|c|c|c|c|c|c|}
\hline \multicolumn{2}{|c|}{ QOF effects beyond mortality } & \multicolumn{5}{|c|}{ How long QOF mortality benefit is sustained if QOF discontinued** } \\
\hline Non-fatal outcomes & Increased drug costs & No waning & 1-year waning & 3-year waning & 5-year waning & 10-year waning \\
\hline Included & Included & $49,362^{*}$ & 51,970 & 57,616 & 63,765 & 81,428 \\
\hline Included & Not included & 48,768 & 51,347 & 56,931 & 63,011 & 80,478 \\
\hline Not included & Included & 80,515 & 84,323 & 92,565 & 101,535 & 127,281 \\
\hline Not included & Not included & 79,657 & 83,424 & 91,575 & 100,446 & 125,907 \\
\hline
\end{tabular}

QOF Quality and Outcomes Framework, QALY quality-adjusted life year

*Base-case scenario: non-fatal outcomes and increased drug costs included and instant changes in the QOF mortality benefit if the QOF is discontinued **In waning scenarios, we assumed linear declines in the QOF mortality benefit from the first year in the model to a time in the future (1, 3, 5 or 10 years from the model start), at which point the mortality benefit from the QOF would equal zero 


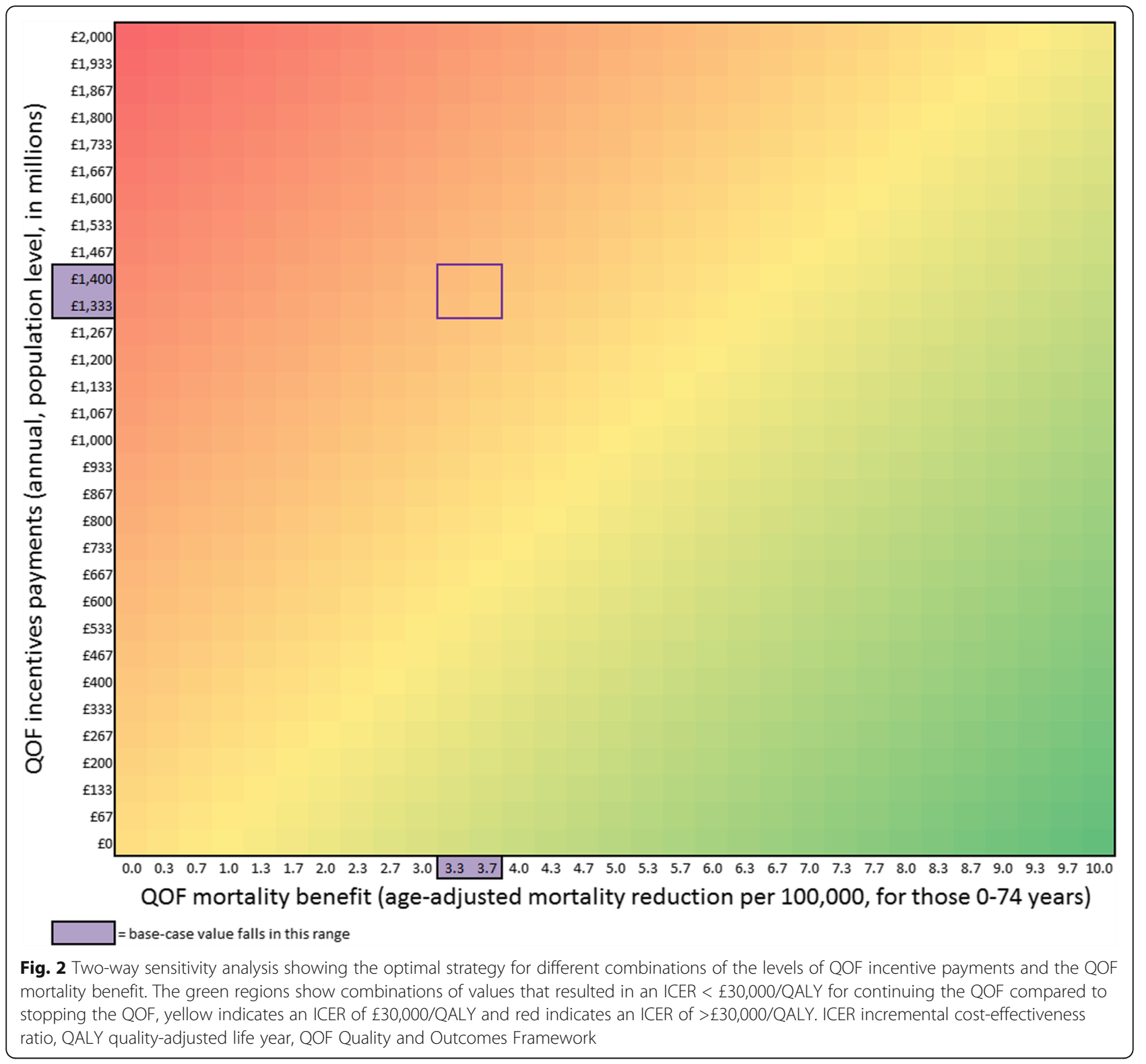

using a cost-effectiveness threshold of $£ 30,000 / Q A L Y$. This estimate was robust to variation in assumptions related to non-fatal outcomes, increased drug costs and waning of benefits from the QOF. A probabilistic sensitivity analysis found that the QOF was cost-effective in only $18 \%$ of the scenarios tested. We found that ICERs of the QOF were substantially more favourable only in those scenarios where the QOF was associated with large reductions (beyond our base-case estimates) in (1) costs associated with averted health events or (2) non-fatal cardiovascular disease events. The estimated population opportunity cost of continuing the QOF (in terms of incremental net health benefit) was 226,109-979,917 QALYs lost.
Our estimate of the ICER for the QOF is above the conventional threshold of $£ 20,000-30,000 / \mathrm{QALY}$ that is used to determine cost-effectiveness in the UK. This suggests that primary-care pay-for-performance in the United Kingdom has not been a cost-effective strategy to improve health. Nonetheless, our base-case analysis treats QOF incentive costs as incremental to the health system. We assumed that stopping the QOF would return all incentive payments to the NHS. However, if the NHS decided to stop the QOF and return all or some of the QOF payments to providers as increased capitation payments, as has already happened in Scotland, this would maintain the costs for the QOF while losing the benefits (unless the benefits from the QOF remain or wane over time after the 


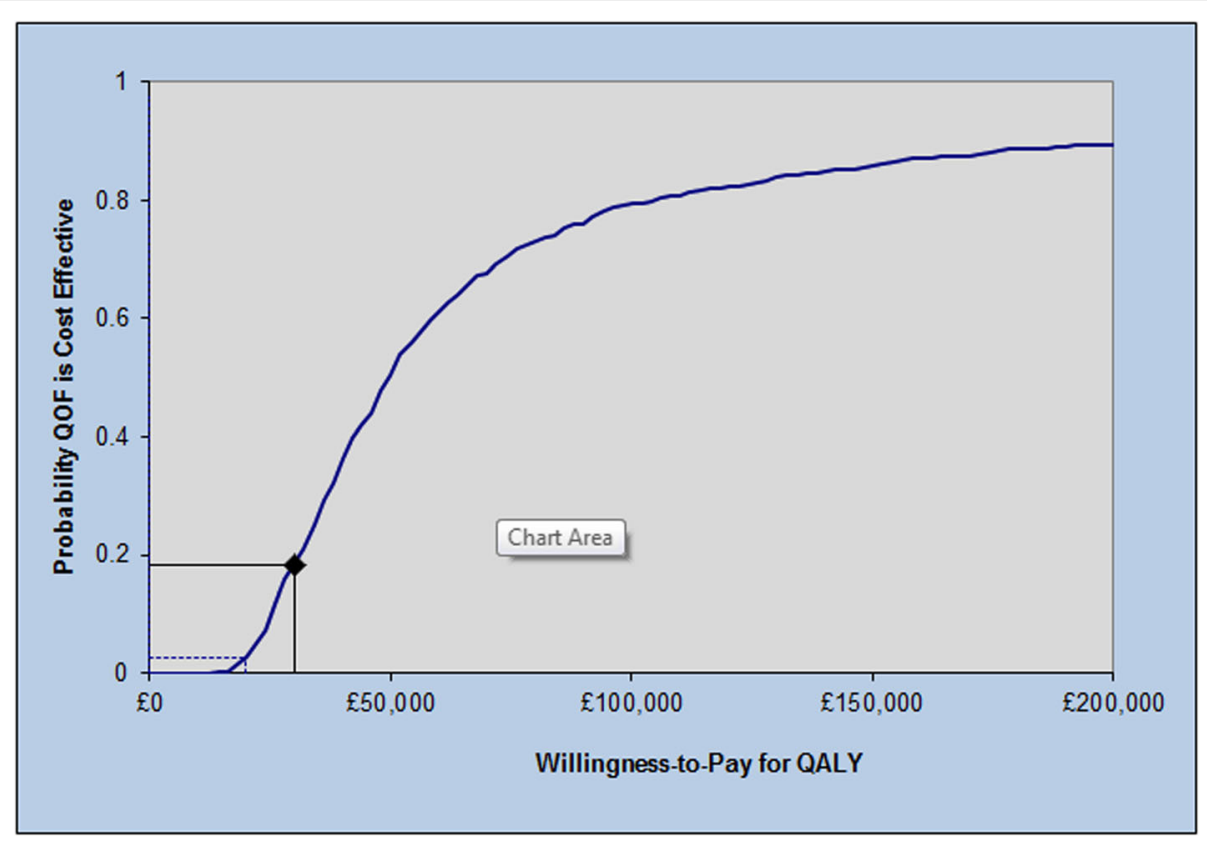

Fig. 3 Cost-effectiveness acceptability curve for the probabilistic sensitivity analysis. The curve shows the probability that the QOF was cost-effective. It was calculated as the proportion of iterations with ICERs that were less than a given cost-effectiveness threshold. The health benefit (base-case value of 3.68 per 100,000 age-adjusted mortality reduction) was randomly drawn from a normal distribution ( $95 \%$ confidence interval -0.80 to 8.16$)$. ICER incremental cost-effectiveness ratio, QALY quality-adjusted life year, QOF Quality and Outcomes Framework

financial incentives are stopped). Relative to this scenario, continuing the QOF would be more favourable. In sensitivity analyses, we found that continuing the QOF would be cost-effective (ICER $<£ 30,000 / \mathrm{QALY}$ or $<£ 13,000$ / QALY) if QOF incentive payments (see Fig. 2) were respectively $32 \%$ or $64 \%$ lower than our base-case estimate, assuming that the mortality impact of QOF remained unchanged. Our analysis gives policymakers cost-effectiveness information for all joint scenarios of QOF payment and mortality benefit estimates to facilitate decision-making around lower QOF payments considering potential reductions in QOF benefits that would follow from these decisions. Our results were not sensitive to the assumption of concentrating the QOF benefits in those with cardiovascular disease, as shown with an ICER > $₫ 30,000 /$ QALY, even if assuming the QOF equally benefited all individuals aged 0-74 years with and without disease.

In general, cost-effectiveness analyses of pay-for-performance policies, especially those that use QALYs as the effectiveness measure (i.e. cost-utility analyses), are rare. In a systematic review of economic evaluations of pay-for-performance policies, Emmert and co-authors found only one cost-utility analysis [10]. That study, by Nahra and co-authors, modelled how hospital process improvements in heart-related care could be used to estimate QALYs via improved medication compliance and found that the pay-for-performance policy was costeffective [13]. Since the publication of the review by
Emmert et al., Meacock et al. and Walker et al. performed cost-utility analyses of pay-for-performance policies. Meacock and colleagues evaluated the cost-effectiveness of a pay-for-performance scheme for hospitals in the UK (the Advancing Quality programme) using reductions in 30-day mortality (among patients admitted for pneumonia, heart failure or acute myocardial infarction) estimated from a difference-in-difference study and found that the programme was cost-effective using a threshold of $£ 20,000 /$ QALY [11]. Walker and co-authors used previously published literature to estimate the potential cost-effectiveness of the QOF. They found that, for most QOF indicators studied, a less than $1 \%$ improvement would be needed for the programme to be cost-effective (using the $£ 20,000-30,000 / Q A L Y$ threshold range). However, this study used estimates from randomised controlled trials to extrapolate the hypothetical effects of incentivising individual activities for which evidence on effectiveness was available, rather than estimating the impact of the overall programme [9].

Our study conflicts with previous attempts to estimate the cost-effectiveness of pay-for-performance policies. Only the Meacock et al. study used effectiveness estimates (reduction in mortality that was translated to QALYs gained) that were directly measured, as opposed to extrapolating intermediate outcomes (such as improvements in medication compliance) to QALYs. The Meacock costeffectiveness study was based on a difference-in-difference 
analysis that found no significant impact of a hospitalbased pay-for-performance programme on outcomes for two of the incentivised conditions (acute myocardial infarction and heart failure) and a modest improvement for the third (pneumonia) after 18 months [36]. However, this improvement was not sustained and re-analysis of the original data using a synthetic control approach found that the initial improvement for pneumonia was not statistically significant $[37,38]$. Unlike the Meacock et al. study, we were not able directly to measure and thus include the administrative costs of running the pay-forperformance programme. Our study is the first to evaluate the cost-effectiveness of the QOF using direct estimates of mortality (as opposed to intermediate outcomes or hospital-based pay-for-performance policies).

Our study has several limitations, including four related to data limitations. First, we could not estimate administrative costs due to data limitations. If administrative cost data become available, these costs could be added to the annual incentive costs in our sensitivity analysis around costs (the horizontal axis in Fig. 2) to estimate the impact of these costs on the cost-effectiveness results. The addition of these administrative costs would increase the ICER for continuing the QOF. Second, although our model cohort was simulated until death, we restricted QOF mortality effects up to age 74 years given the source data [6]. Third, we also had limited information on the effect of the QOF on costs, such as those of additional visits to practices, referrals to secondary care and medication prescriptions. For instance, there are very few studies estimating the impact of the QOF on health-care utilisation, which is why we relied on a 2008 observational study from Scotland to estimate incentivised drug costs despite our model assuming a causal relationship between the QOF incentives and increased utilisation. To address this, we performed a sensitivity analysis around this input value, which showed that cost savings because of the QOF (i.e. averted utilisation costs outweighing the incentive costs) would be necessary to make the QOF cost-effective using a threshold of $£ 30,000 / Q A L Y$. Fourth, we also had incomplete information about the effects of the QOF on nonfatal outcomes, such as acute myocardial infarction and stroke. To address this, we varied our estimates across a range of assumptions about the effects of the QOF non-fatal outcomes and found that the ratio of fatal-tonon-fatal events would need to be more than doubled from our base-case estimate to make the QOF cost-effective. Fifth, our results from a probabilistic sensitivity analysis showed that there is considerable statistical uncertainty around the cost-effectiveness of continuing the QOF, suggesting that more precise estimates of the effect of the QOF on mortality could reduce the uncertainty around the decision of whether to continue the QOF. We also varied only one parameter (the effectiveness of the
QOF) in the probabilistic sensitivity analysis because it was the only input with a well-estimated $95 \%$ confidence interval. Adding other parameters to the probabilistic sensitivity analysis could produce a higher uncertainty in our cost-effectiveness results, but that would not change the overall conclusion of our analysis. Sixth, QOF is subject to annual review and amendment $[6-8,24,26]$, and our main estimates for the effectiveness and incentives costs were based on the first 7 years of the programme. Therefore, our analysis is evaluating the decision to continue with incentives contained in QOF from 2004 to 2010 versus discontinuing these incentives. We have not evaluated the most recent versions of the QOF, for which we do not have linked cost and effectiveness data, but incentives for the conditions of interest were retained in these versions.

Despite these limitations, our findings imply that the UK should redesign the QOF or pursue alternative interventions to improve population health efficiently. The QOF is already in transition. The programme was reduced in scope in 2014, with 40 indicators retired to focus on a set of 83 key indicators [7, 39]. In Scotland, the QOF was withdrawn altogether in 2016, with practices continuing to receive payments based on their historical performance without any further need to meet QOF targets. In the future, quality improvement in Scottish practices will be managed by local peer support networks and will rely on clinical governance arrangements rather than financial incentives [40]. NHS England is also now seeking to develop a successor to the QOF [41]. To enable informed decisions on further redesign or replacement of the QOF, future research should compare the costeffectiveness of the programme with alternative systemlevel interventions (whether these programmes are different forms of financial incentives for providers or patients, or use other mechanisms to improve quality, costs or access to care). Similar research should be undertaken in other settings where pay-for-performance has been implemented, comparing its cost-effectiveness with other health system-level policies such as value-based insurance design [42], computerised decision support interventions [43] or value-based outcome reporting tools [44]. These future analyses would provide crucial information about whether pay-for-performance in primary care is a cost-effective way to improve population health.

\section{Conclusions}

Compared to stopping the QOF and returning all associated incentive payments to the NHS, continuing the QOF is not cost-effective. To improve population health efficiently, the UK should redesign the QOF or pursue alternative interventions. 


\section{Appendix}

Table 4 Estimates of incentive payments to UK practices under the Quality and Outcomes Framework (inflated to 2016£)

\begin{tabular}{llllll}
\hline Year & England $^{\mathrm{a}}$ & Northern Ireland & Scotland $^{\mathrm{b}}$ & Wales $^{\mathrm{a}}$ & Total $^{\mathrm{a}}$ \\
\hline $2004 / 5$ & $£ 830,220,692$ & $£ 36,069,236$ & $£ 90,923,805$ & $£ 48,008,831$ & $£ 1,005,222,565$ \\
$2005 / 6$ & $£ 1,350,199,371$ & $£ 58,115,719$ & $£ 155,248,597$ & $£ 79,707,784$ & $£ 1,643,271,471$ \\
$2006 / 7$ & $£ 1,237,234,310$ & $£ 53,602,369$ & $£ 144,140,152$ & $£ 73,024,636$ & $£ 1,508,001,467$ \\
$2007 / 8$ & $£ 1,213,821,483$ & $£ 52,481,420$ & $£ 142,189,299$ & $£ 72,081,589$ & $£ 1,480,573,791$ \\
$2008 / 9$ & $£ 1,147,372,625$ & $£ 49,335,331$ & $£ 135,246,153$ & $£ 67,898,096$ & $£ 1,399,852,206$ \\
$2009 / 10$ & $£ 1,128,185,395$ & $£ 48,508,555$ & $£ 132,825,069$ & $£ 66,939,222$ & $£ 1,376,458,241$ \\
$2010 / 11$ & $£ 1,122,907,420$ & $£ 46,715,424$ & $£ 128,483,891$ & $£ 66,415,581$ & $£ 1,364,522,315$ \\
Total & $£ 8,029,941,295$ & $£ 344,828,055$ & $£ 929,056,966$ & $£ 474,075,739$ & $£ 9,777,902,056$ \\
\hline
\end{tabular}

${ }^{\mathrm{a}}$ Based on estimates from the Information Centre for Health and Social Care $[24,26]$

based on payment data from the Information Services Division Scotland [27]

Table 5 Drug classes, changes in utilisation and annual prices

\begin{tabular}{lll}
\hline Drug class ${ }^{\mathrm{a}}$ & DDD/PU/mo & Annual price $^{\mathrm{b}}$ \\
\hline Lipid regulating drugs & 1.92 & 61.57 \\
Renin angiotensin & 0.84 & 37.29 \\
Thiazides/diuretics & 0.24 & 5.66 \\
Oral antidiabetic & 0.14 & 42.79 \\
Antiplatelet & 0.33 & 14.77
\end{tabular}

$D D D$ defined daily dose, mo month, $P U$ prescribing unit, QOF Quality and Outcomes Framework

${ }^{a}$ Cardiovascular disease drug classes from MacBride-Stewart et al. [29]

${ }^{b}$ Increase in defined daily doses (DDDs) per prescribing unit (PU) per month of QOF-related drugs

'Weighted average price (weights based on quantity dispensed by specific drugs and doses)

(source: Health and Social Care Information Centre. Prescription cost analysis, England 2012)

https://digital.nhs.uk/data-and-information/publications/statistical/prescription-cost-analysis/prescription-cost-analysis-england-2012

Table 6 Population-level incremental net health benefit results

\begin{tabular}{|c|c|c|c|c|c|}
\hline & \multirow[b]{2}{*}{ Discounted QALYs } & \multirow[b]{2}{*}{ Discounted costs } & \multicolumn{3}{|c|}{ Net health benefit using different cost-effectiveness thresholds (QALYs) } \\
\hline & & & $£ 30,000 / \mathrm{QALY}$ threshold & $£ 20,000 / Q A L Y$ threshold & $£ 13,000 /$ QALY threshold \\
\hline Stopping the QOF & $39,966,375$ & $£ 0$ & $39,966,375$ & $39,966,375$ & $39,966,375$ \\
\hline Continuing the QOF & $40,316,707$ & $£ 17,293,239,670$ & $39,740,266$ & $39,452,046$ & $38,986,458$ \\
\hline Delta & 350,332 & $£ 17,293,239,670$ & $-226,109$ & $-514,330$ & $-979,917$ \\
\hline
\end{tabular}

QOF Quality and Outcomes Framework, QALY quality-adjusted life year

Table 7 One-way sensitivity analysis results

\begin{tabular}{|c|c|c|c|c|}
\hline Variable & Base-case value & Sensitivity analysis range & ICER at low value & ICER at high value \\
\hline $\begin{array}{l}\text { Adjusted QOF mortality benefit (for those aged }>40 \text { years } \\
\text { with CVD) }\end{array}$ & 58.93 & -130.57 to 12.81 & QOF dominated & $£ 20,044 / \mathrm{QALY}$ \\
\hline CVD prevalence, males (aged 45-64 years, aged 65-74 years) & $14.6 \%, 28.5 \%$ & $\pm 20 \%$ & $£ 49,266 / \mathrm{QALY}$ & $£ 49,485 / \mathrm{QALY}$ \\
\hline CVD prevalence, females (aged 45-64 years, aged 65-74 years) & $8.4 \%, 22.5 \%$ & $\pm 20 \%$ & $£ 49,302 / \mathrm{QALY}$ & $£ 49,414 / \mathrm{QALY}$ \\
\hline CVD mortality multiplier (male, female) & $1.6,2.1$ & $\pm 20 \%$ & $£ 47,246 / \mathrm{QALY}$ & $£ 51,249 / \mathrm{QALY}$ \\
\hline CVD utility & 0.796 & $\pm 20 \%$ & $£ 43,516 / \mathrm{QALY}$ & $£ 64,805 / \mathrm{QALY}$ \\
\hline Acute CVD event costs (i.e. costs within first year of CVD event) & $£ 10,871$ & $\pm 20 \%$ & $£ 49,850 / Q A L Y$ & $£ 48,874 / \mathrm{QALY}$ \\
\hline $\begin{array}{l}\text { Chronic CVD event costs (i.e. annual costs for all years after } \\
\text { first year) }\end{array}$ & $£ 3282$ & $\pm 20 \%$ & $£ 50,350 / \mathrm{QALY}$ & $£ 48,375 / \mathrm{QALY}$ \\
\hline Average NHS costs by age & Age-based table & $£ 0$ to $+100 \%$ & $£ 46,270 / \mathrm{QALY}$ & $£ 52,455 / \mathrm{QALY}$ \\
\hline Discount rate & $3.5 \%$ & $0-5 \%$ & $£ 38,337 / \mathrm{QALY}$ & $£ 54,587 / \mathrm{QALY}$ \\
\hline
\end{tabular}

Base-case ICER of $£ 49,362 / \mathrm{QALY}$; base-case values for each variable are reported in Table 1 in the main text

CVD cardiovascular disease, ICER incremental cost-effectiveness ratio, NHS National Health Service, QALY quality-adjusted life year, QOF Quality and Outcomes Framework 


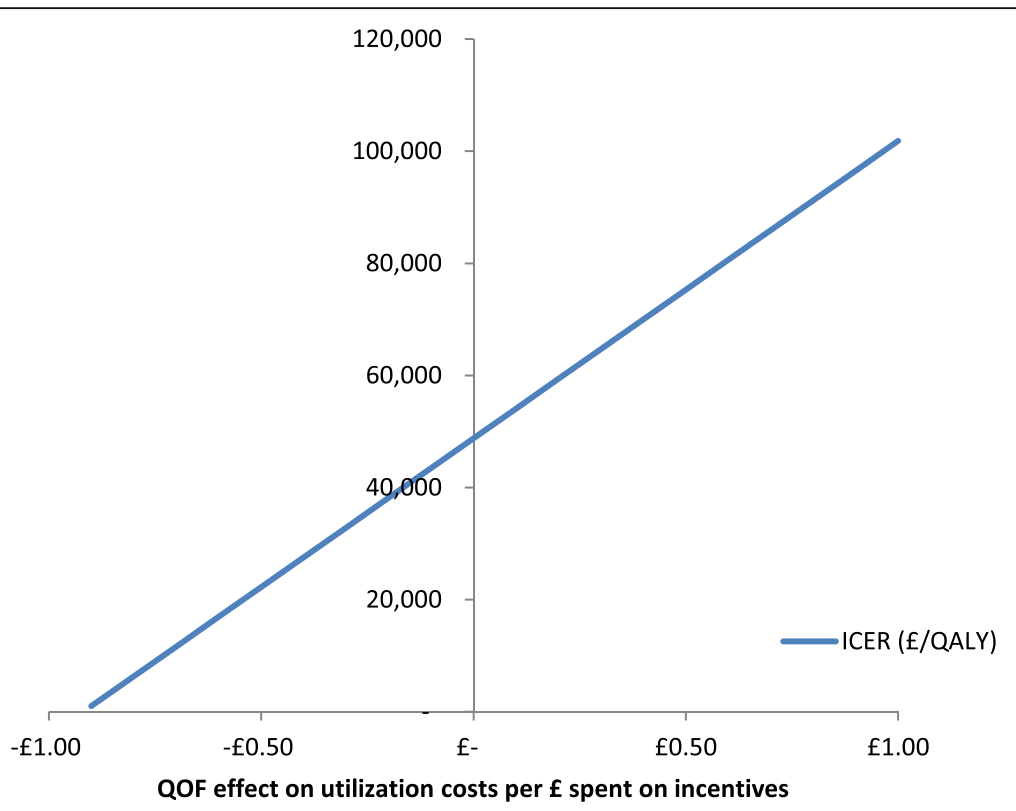

Fig. 4 Cost-effectiveness ratio ( $£ /$ QALY) for the QOF versus non-QOF as a function of QOF-incentivised utilisation costs. ICER incremental costeffectiveness ratio, QALY quality-adjusted life year, QOF Quality and Outcomes Framework

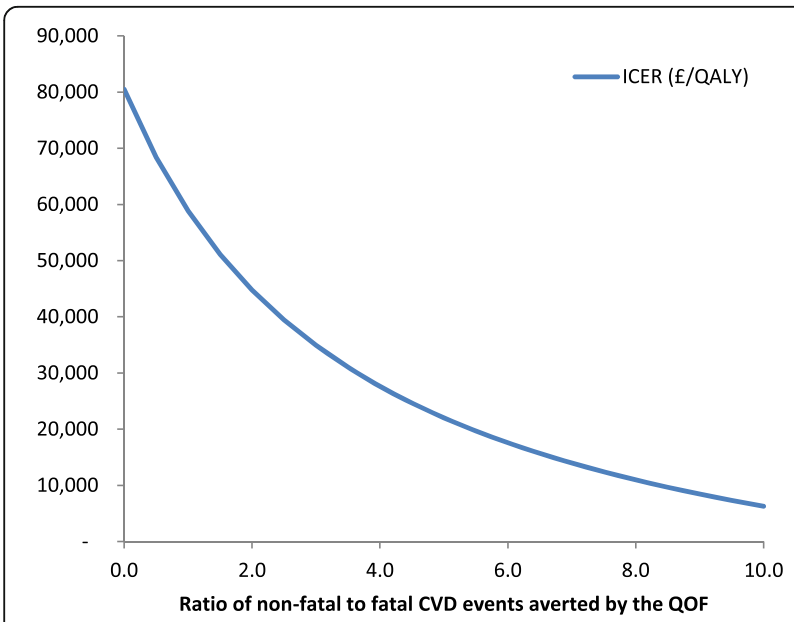

Fig. 5 Cost-effectiveness ratio ( $£ / Q A L Y)$ for the QOF versus non-QOF as a function of non-fatal-to-fatal CVD events averted. CVD cardiovascular disease, ICER incremental cost-effectiveness ratio, QALY qualityadjusted life year, QOF Quality and Outcomes Framework 


\section{Additional file}

Additional file 1: The model, which is the full simulation model used to perform the cost-effectiveness analyses, is programed in Microsoft Excel with Visual Basic for Applications (VBA). (XLSM 648 kb)

\section{Abbreviations}

BHF: British heart Foundation; CEAC: Cost-effectiveness acceptability curve; DDD: Defined daily dose; EQ-5D: EuroQOL 5 dimensions; ICD: International classification of diseases; ICER: Incremental cost-effectiveness ratio; NICE: National Institute for health and care excellence; NHS: National health service; PU: Prescribing unit; QALY: Quality-adjusted life year; QOF: Quality and Outcomes Framework; UK: United Kingdom; US: United States

\section{Availability of data and materials}

We have provided the full simulation model used to perform all of our analyses.

\section{Authors' contributions}

AP, TD and AMR conceived the idea of the study. AP, TD, JZ and SW developed the simulation model and estimated model inputs. AP and JZ performed the model-based analyses. AP, EA and AMR wrote the first draft of the manuscript. All authors read and approved the final manuscript.

\section{Ethics approval and consent to participate}

Not applicable.

\section{Consent for publication}

Not applicable.

\section{Competing interests}

The authors declare that they have no competing interests.

\section{Publisher's Note}

Springer Nature remains neutral with regard to jurisdictional claims in published maps and institutional affiliations.

\section{Author details}

'Department of Health Policy and Management, Harvard T.H. Chan School of Public Health, 718 Huntington Ave, 2nd Floor, Boston, MA 02115, USA. ${ }^{2}$ Department of Health Sciences, University of York, Heslington, York, UK. ${ }^{3}$ Center for Health Decision Science, Harvard T.H. Chan School of Public Health, Boston, MA, USA. ${ }^{4}$ Centre for Health Economics, University of York, Heslington, York, UK. ${ }^{5}$ Department of Health Management and Policy, University of Michigan School of Public Health, Ann Arbor, MI, USA

\section{Received: 24 January 2018 Accepted: 12 July 2018}

Published online: 29 August 2018

\section{References}

1. Roland M. Linking physicians' pay to the quality of care--a major experiment in the United Kingdom. N Engl J Med. 2004;351(14):1448-54.

2. Doran T, Kontopantelis E, Valderas JM, Campbell S, Roland M, Salisbury C, Reeves D. Effect of financial incentives on incentivised and non-incentivised clinical activities: longitudinal analysis of data from the UK quality and outcomes framework. BMJ. 2011:342:d3590.

3. Campbell SM, Reeves D, Kontopantelis E, Sibbald B, Roland M. Effects of pay for performance on the quality of primary care in England. N Engl J Med. 2009;361(4):368-78.

4. Fleetcroft R, Parekh-Bhurke S, Howe A, Cookson R, Swift L, Steel N. The UK pay-for-performance programme in primary care: estimation of population mortality reduction. Br J Gen Pract. 2010;60(578):e345-52.

5. Gillam SJ, Siriwardena AN, Steel N. Pay-for-performance in the United Kingdom: impact of the quality and outcomes framework: a systematic review. Ann Fam Med. 2012;10(5):461-8.

6. Ryan AM, Krinsky S, Kontopantelis E, Doran T. Long-term evidence for the effect of pay-for-performance in primary care on mortality in the UK: a population study. Lancet. 2016;388(10041):268-74.
7. Changes to QOF 2014/15: NHS Employers; 2017. http://www.nhsemployers, org/your-workforce/primary-care-contacts/general-medical-services/qualityand-outcomes-framework/changes-to-qof-2014-15.

8. Scotland first to abolish bureaucratic system of GP payments. Government S. 2015. https://news.gov.scot/news/scotland-first-to-abolishbureaucratic-system-of-gp-payments.

9. Walker S, Mason AR, Claxton K, Cookson R, Fenwick E, Fleetcroft R, Sculpher M. Value for money and the quality and outcomes framework in primary care in the UK NHS. Br J Gen Prac. 2010:60(574):e213-20.

10. Emmert M, Eijkenaar F, Kemter $\mathrm{H}$, Esslinger AS, Schoffski O. Economic evaluation of pay-for-performance in health care: a systematic review. Eur J Health Econ. 2012;13(6):755-67.

11. Meacock R, Kristensen SR, Sutton M. The cost-effectiveness of using financia incentives to improve provider quality: a framework and application. Health Econ. 2014:23(1):1-13.

12. Eijkenaar F, Emmert M, Scheppach M, Schoffski O. Effects of pay for performance in health care: a systematic review of systematic reviews. Health Policy. 2013;110(2-3):115-30.

13. Nahra TA, Reiter KL, Hirth RA, Shermer JE, Wheeler JR. Cost-effectiveness of hospital pay-for-performance incentives. Med Care Res Rev. 2006;63(1 Suppl):49S-72S.

14. Guide to the methods of technology appraisal. National Institute for Health and Care Excellence. 2013. https://www.nice.org.uk/process/pmg9/resources/ guide-to-the-methods-of-technology-appraisal-2013-pdf-2007975843781.

15. Population Estimates for UK, England and Wales, Scotland and Northern Ireland: Office for National Statistics. 2016.https://www.ons.gov.uk/ peoplepopulationandcommunity/populationandmigration/ populationestimates/datasets/ populationestimatesforukenglandandwalesscotlandandnorthernireland.

16. Cardiovascular Disease Statistics 2015. British Heart Foundation. 2015.https:// www.bhf.org.uk/informationsupport/publications/statistics/cvd-stats-2015.

17. Doran T, Fullwood C, Gravelle H, Reeves D, Kontopantelis E, Hiroeh U, Roland M. Pay-for-performance programs in family practices in the United Kingdom. N Engl J Med. 2006:355(4):375-84.

18. Smolina K, Wright FL, Rayner M, Goldacre MJ. Long-term survival and recurrence after acute myocardial infarction in England, 2004 to 2010. Cir Cardiovas Qual Outcomes. 2012;5(4):532-40.

19. Roberts M, Russell LB, Paltiel AD, Chambers M, McEwan P, Krahn M. Conceptualizing a model: a report of the ISPOR-SMDM modeling good research practices task Force-2. Med Decis Mak. 2012;32(5):678-89.

20. Baigent C, Keech A, Kearney PM, Blackwell L, Buck G, Pollicino C, Kirby A, Sourjina T, Peto R, Collins R, et al. Efficacy and safety of cholesterol-lowering treatment: prospective meta-analysis of data from 90,056 participants in 14 randomised trials of statins. Lancet. 2005;366(9493):1267-78.

21. Sullivan PW, Ghushchyan V. Preference-based EQ-5D index scores for chronic conditions in the United States. Med Decis Mak. 2006;26(4):410-20.

22. Dolan P. Modeling valuations for EuroQol health states. Med Care. 1997 35(11):1095-108

23. Sullivan PW, Slejko JF, Sculpher MJ, Ghushchyan V. Catalogue of EQ-5D scores for the United Kingdom. Med Decis Mak. 2011;31(6):800-4.

24. Investment in General Practice 2003/04 to 2007/08 England, Wales, and Northern Ireland. National Health Service. 2009. http://webarchive. nationalarchives.gov.uk/20180328130852tf/http://content.digital.nhs.uk/ catalogue/PUB01074/inve-gene-prac-eng-wal-ni-03-08-rep.pdf/.

25. Roland M, Guthrie B. Quality and outcomes framework: what have we learnt? BMJ. 2016:354:14060.

26. Investment in General Practice 2008/09 to 2012/13 England, Wales, Northern Ireland and Scotland. National Health Service. 2013. http://webarchive nationalarchives.gov.uk/20180328130852tf_/http://content.digital.nhs.uk/ catalogue/PUB11679/inve-gene-prac-eng-wal-ni-scot-08-13.pdf/.

27. Quality and Outcomes Framework. Information Services Division Scotland. 2016. http://www.isdscotland.org/health-Topics/GeneralPractice/Quality-And-Outcomes-Framework/.

28. Doran T, Roland M. Lessons from major initiatives to improve primary care in the United Kingdom. Health Aff (Millwood). 2010;29(5):1023-9.

29. MacBride-Stewart SP, Elton R, Walley T. Do quality incentives change prescribing patterns in primary care? An observational study in Scotland. Fam Pract. 2008:25(1):27-32

30. Department of Health. NHS reference costs: financial year 2011 to 2012. 2012.

31. Walker S, Asaria M, Manca A, Palmer S, Gale CP, Shah AD, Abrams KR, Crowther M, Timmis A, Hemingway $H$, et al. Long-term healthcare use and 
costs in patients with stable coronary artery disease: a population-based cohort using linked health records (CALIBER). Eur Heart J Qual Care Clin Outcomes. 2016;2(2):125-40.

32. Asaria M. Health Care Costs in the English NHS: reference tables for average annual NHS. https://www.york.ac.uk/media/che/documents/papers/ researchpapers/CHERP147_health_care_costs_NHS.pdf.

33. Claxton K, Martin S, Soares M, Rice N, Spackman E, Hinde S, Devlin N, Smith PC, Sculpher M. Methods for the estimation of the National Institute for health and care excellence cost-effectiveness threshold. Health Technol Assess. 2015;19(14):1-503. v-vi

34. Stinnett AA, Mullahy J. Net health benefits: a new framework for the analysis of uncertainty in cost-effectiveness analysis. Med Decis Mak. 1998; 18(2 Suppl):S68-80.

35. Doubilet $P$, Begg CB, Weinstein MC, Braun P, McNeil BJ. Probabilistic sensitivity analysis using Monte Carlo simulation. A practical approach. Med Decis Making. 1985;5(2):157-77.

36. Sutton M, Nikolova S, Boaden R, Lester H, McDonald R, Roland M. Reduced mortality with hospital pay for performance in England. N Engl J Med. 2012; 367(19):1821-8.

37. Kreif N, Grieve R, Hangartner D, Turner AJ, Nikolova S, Sutton M Examination of the synthetic control method for evaluating health policies with multiple treated units. Health Econ. 2016;25(12):1514-28.

38. Kristensen SR, Meacock R, Turner AJ, Boaden R, McDonald R, Roland M, Sutton M. Long-term effect of hospital pay for performance on mortality in England. N Engl J Med. 2014;371(6):540-8.

39. Doran T, Kontopantelis E, Reeves D, Sutton M, Ryan AM. Setting performance targets in pay for performance programmes: what can we learn from QOF? Bmj. 2014;348:g1595.

40. Improving together. A national framework for quality and GP clusters in Scotland. Scottish Government. 2017. http://www.gov.scot/Resource/0051/ 00512739.pdf.

41. NHS five year forward view: national health service England; 2017.https:// www.england.nhs.uk/five-year-forward-view/next-steps-on-the-nhs-five-yearforward-view/primary-care/.

42. Braithwaite RS, Omokaro C, Justice AC, Nucifora K, Roberts MS. Can broader diffusion of value-based insurance design increase benefits from US health care without increasing costs? Evidence from a computer simulation model. PLoS Med. 2010;7(2):e1000234.

43. O'Reilly D, Holbrook A, Blackhouse G, Troyan S, Goeree R. Cost-effectiveness of a shared computerized decision support system for diabetes linked to electronic medical records. J Am Med Inform Assoc. 2012;19(3):341-5.

44. Lee VS, Kawamoto K, Hess R, Park C, Young J, Hunter C, Johnson S, Gulbransen S, Pelt CE, Horton DJ, et al. Implementation of a value-driven outcomes program to identify high variability in clinical costs and outcomes and association with reduced cost and improved quality. JAMA. 2016:316(10):1061-72.

45. National jife tbles. United Kingdom: Office for national statistics; 2016. https://www.ons.gov.uk/peoplepopulationandcommunity/ birthsdeathsandmarriages/lifeexpectancies/datasets/ nationallifetablesunitedkingdomreferencetables.

Ready to submit your research? Choose BMC and benefit from:

- fast, convenient online submission

- thorough peer review by experienced researchers in your field

- rapid publication on acceptance

- support for research data, including large and complex data types

- gold Open Access which fosters wider collaboration and increased citations

- maximum visibility for your research: over $100 \mathrm{M}$ website views per year

At BMC, research is always in progress.

Learn more biomedcentral.com/submissions 